\title{
Dynamic response investigation of dynamic cable in wave energy generator under shallow water and severe sea conditions
}

\author{
Dahui Liu', Yao Teng1, 2, *, Hui Zhao ${ }^{1}$, Shoujun Wang', 2, Yuxiang Niü ${ }^{3}$ and Wanqing Zhang \\ ${ }^{1}$ CIMC Offshore Engineering Institute Co., Ltd., Yantai, China \\ ${ }^{2}$ College of Power and Energy Engineering, Harbin Engineering University, Harbin, China \\ ${ }^{3}$ College of Shipbuilding Engineering, Harbin Engineering University, Harbin, China \\ *Corresponding author: daweiping@126.com
}

Keywords: shallow water condition, wave energy, dynamic cable, effective tension, bending curvature.

\begin{abstract}
Wave energy is an important clean renewable energy source for the ocean, and is of great importance for development. However, it is difficult to achieve the unity of high efficiency and high reliability of existing wave energy development devices. In this paper, a new form of fully enclosed eccentric pendulum wave energy generation device is proposed. A hydrodynamic model based on AQWA software, a mechanism dynamics model based on Adams software, and a hydraulic energy conversion system model based on AMESim software are developed to analyze the motion response and power generation performance of the device under different wave excitation conditions. The results show that the wave excitation conditions and the rotor eccentricity jointly affect the power generation performance of the wave energy generation device, and the high efficiency of wave energy capture can be obtained by real-time adjustment of the rotor eccentricity. This paper both exploits the high reliability of the fully enclosed structure and proposes a control strategy to achieve efficient energy conversion, laying the foundation for the development of a wave energy generation device with both high efficiency and high reliability.
\end{abstract}

\section{Introduction}

Since the 21st century, the reserves of traditional energy resources represented by fossil energy have been declining day by day. And it is increasingly difficult to satisfy the energy consumption with human social's economic development and civilization progress. In addition, these traditional sources of energy will produce a number of environmental pollution during its use and consumption. These pollutants may directly damage human's health under the background of serious environmental pollution presently. In order to solve this energy dilemma effectively, more attention will be paid to the development and utilization of clean and renewable energy. Especially the development and utilization of wind power which is non-polluting and widely distributed will be focused on. Among them, wave power generation has the advantages of pollution-free, renewable, large reserves, wide distribution and so on. Compared with other clean energy sources such as solar energy and wind energy, wave power generation also has the characteristics of high density. China has a vast coastline and numerous island resources, the wave energy resources are extremely rich. As China puts forward the goal of "carbon peak and carbon neutral", wave power generation has great commercial prospects and it will attracts more and more scholars and engineers to carry out research.

According to the working principle of hydrodynamic subsystem, wave energy devices can be roughly divided into three categories: (1) oscillating water column wave energy generator, (2) wavetype wave energy generator, (3) oscillating float type wave energy generator. The wave energy device captures the wave energy through a mechanical energy capture mechanism, and then uses the energy conversion and transfer system to transfer, store and transform the captured energy, and finally outputs it in the form of electric energy by dynamic cable (Shi et al., 2021) [1]. At present, the research on wave energy devices and the motion response and energy conversion efficiency is getting more 
sophisticated (Mosquera et al., 2020; Roy, 2021) [2,3,4,5]. Tian et al. (2021) from Wuhan University of Technology developed a device of two-body floating point absorber with retractable power takeoff, and the feasibility and efficiency of the power generation of the device were verified through flume test [2]. Zhang et al. (2021) from Guangzhou Institute of Energy Resources, Chinese Academy of Sciences, based on the wave energy characteristics of the Marine environment in Wanshan sea area, Zhuhai, where the eagle wave power generation device was launched, found that the combination mode with a wave retaining wall and a wave guide bulge has the advantage of high capture efficiency and self-protection function under extreme sea conditions [3]. Xu et al. (2020) from South China University of Technology adopted AQWQ software to establish a computational model of the floattype power generation device to study the hydrodynamic performance and initial energy conversion efficiency of the multi-degree-of-freedom wave energy conversion device, and they found that heave motion was the main source of energy absorption of the float-type power generation device [4]. Most of the above studies take wave energy devices as the research object, but there are not sufficient reports on the dynamic cable (see Figure 1) used for the power transmission of wave energy generation devices. In this paper, the dynamic cable of built-in eccentric rotor wave power generator is taken as the research object. Based on the AQWA module, a numerical simulation model of the wave energy generation device with built-in eccentric rotor is established to explore the motion response of built-in eccentric rotor wave power generator under the action of three cables mooring. The motion response of wave power generation device simulated by AQWA was taken as the motion response of dynamic cable top-end, and the motion responses of different dynamic cable configurations under harsh conditions in shallow water were compared.

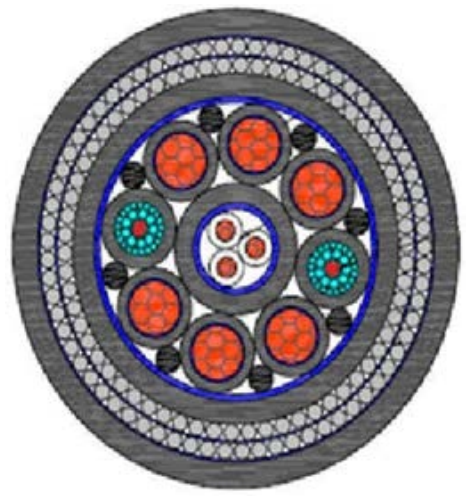

Figure 1. Typical section for dynamic cable

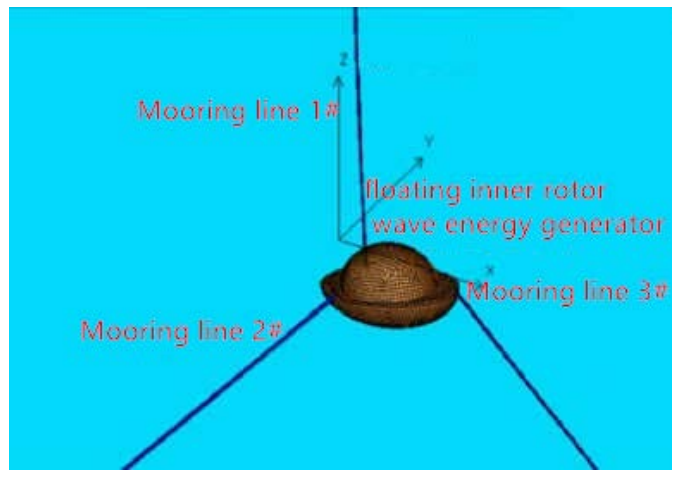

Figure 2. Analysis model of wave power generation device in AQWA

\section{Motion analysis of wave power generation device based on AQWA}

As shown in Figure 2, the numerical simulation model of the wave energy generator with built-in eccentric rotor wave power generator was established by ANSYS AQWA software. Among them, the lower shell of wave energy generating device is a semi-ellipsoid with a long axis of $2 \mathrm{~m}$, a short axis of $1.1 \mathrm{~m}$ and a height of $0.55 \mathrm{~m}$. The designed wave power generator has a total mass of $464 \mathrm{~kg}$ and a draft of $0.45 \mathrm{~m}$. Three cable moorings are employed and the initial set mooring chain diameter was $11 \mathrm{~mm}$. By setting different wind and wave conditions, the motion of the floating body for built-in eccentric rotor wave power generator was analyzed under different sea conditions. In this paper, the numerical simulation model was conducted based on AQWA software. In view of the paper length limitation, one of the environmental conditions is used for numerical simulation, and the specific environmental parameters are as follows: (1) JONSWAP spectrum, with significant wave height $1.5 \mathrm{~m}$ and zero-crossing period 6.0s. (2) Current velocity $0.88 \mathrm{~m} / \mathrm{s}$. (3) Wind velocity $8 \mathrm{~m} / \mathrm{s}$. The analysis time is set to 1000s, and the motion time history curve of the wave power generator in six degrees of freedom is shown in Figure 3. 

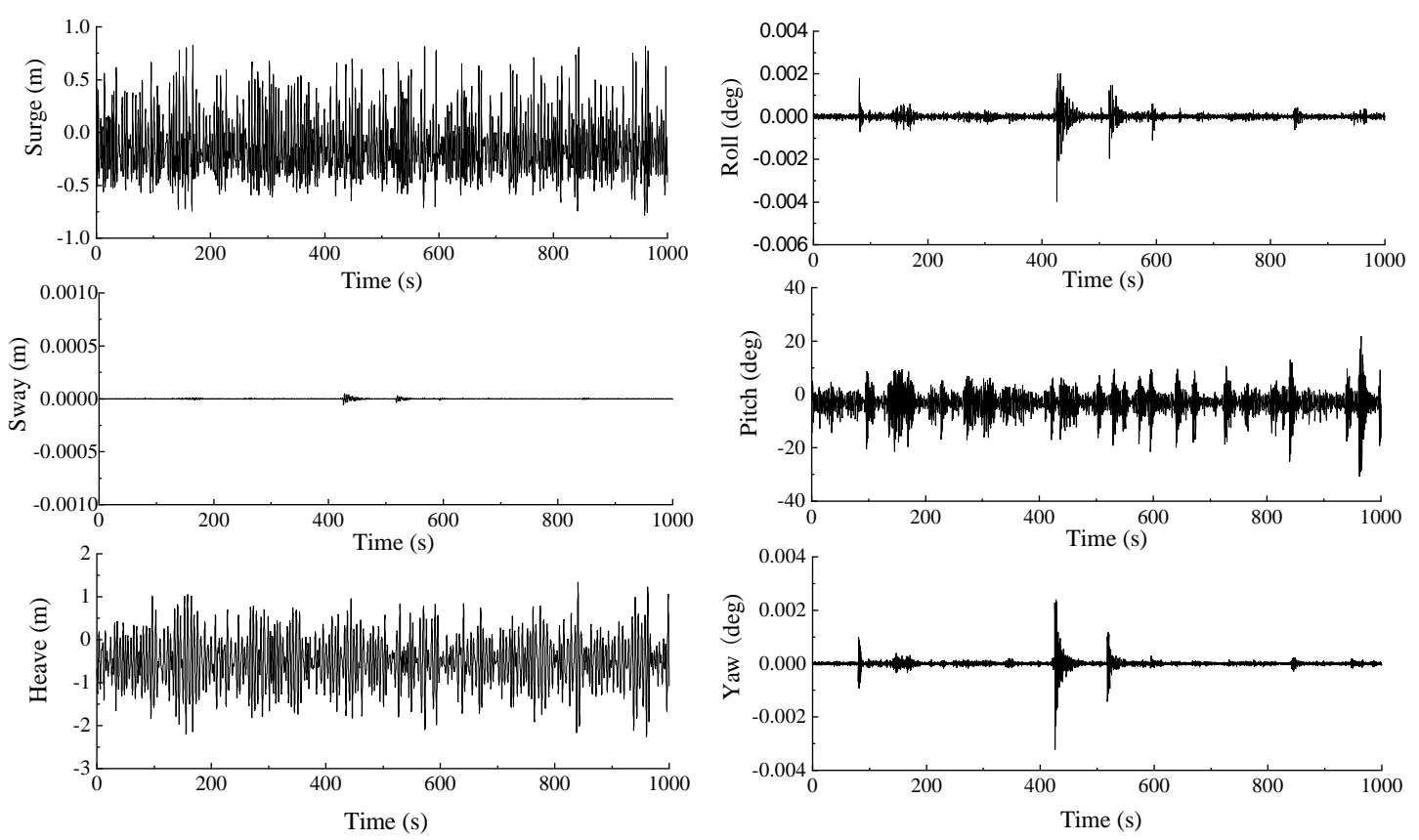

Figure 3. Six degrees of freedom motion response of wave energy generator

\section{Dynamic cable motion analysis based on OrcaFlex}

In this paper, OrcaFlex software is employed as the finite element numerical simulation platform to establish the numerical simulation model for the dynamic cable dynamic response of built-in eccentric rotor wave power generator in three-dimensional space. The model adopts JONSWAP spectrum and shear flow profiles to simulate wave and constant flows in different directions, and the wave spectrum equation can be described as:

$$
S(w)=\left(\frac{\alpha g^{2}}{\omega^{5}}\right) \exp \left[-1.25\left(\frac{\omega_{p}}{\omega}\right)^{4}\right] \gamma^{b}
$$

where: $\alpha$ is the Philips constant, $g$ is the gravitational acceleration, $\omega$ is the circular frequency, $\omega_{p}$ is the peak frequency, $\gamma$ is the JONSWAP peak state parameter, $b=\exp \left\{-\left(\omega_{-} \omega_{p}\right)^{2} / 2 \sigma^{2} \omega_{p}{ }^{2}\right\}, \sigma$ is the number of spectral width parameters.
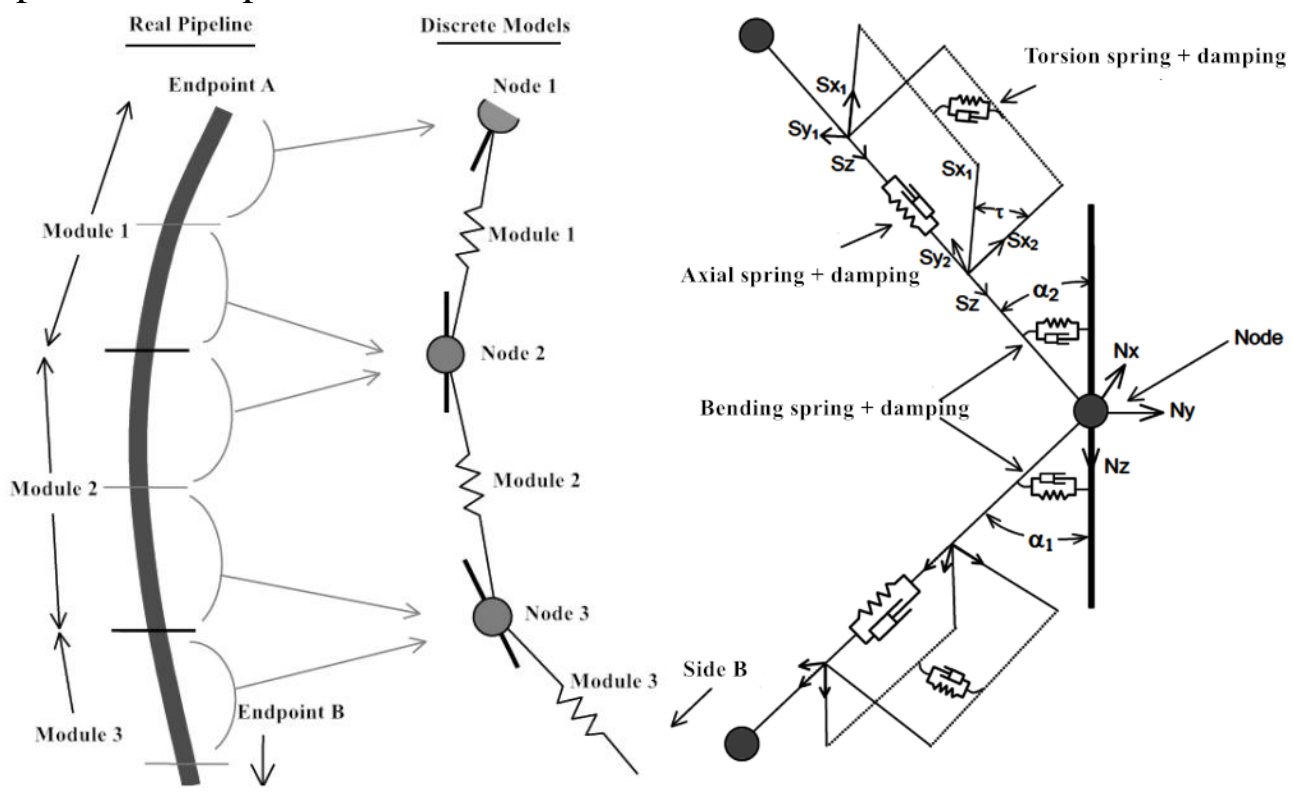

Figure 4. OrcaFlex software "Line" module model (Orcina, 2017) 
As a typical marine slender structure, the marine dynamic cable is simulated by using Line module, which is discretized by OrcaFlex software based on the lumped-mass method into a number of springconnected massless continuous elements (the springs connected between the nodes include axial tension spring, bending spring, torsion spring and damper). The model simulates the mechanical and deformation characteristics of a dynamic cable by means of element forces and spring deformations (Figure 4). The effective tension Te for the cable under hydrostatic internal and external pressure is calculated as follows [7]:

$$
T_{e}=T_{w}+(1-2 v)\left(P_{o} A_{o}-P_{i} A_{i}\right)+E A e(d L / d t) / L_{0}
$$

where Tw is the wall tension, $v$ is the Poisson's ratio, Ai and Ao are the internal and external crosssectional areas of dynamic cable, respectively, Pi and Po are the internal and external hydrostatic pressures, respectively, EA is the axial stiffness of the cable, e is the damping coefficient of the cable, $\mathrm{dL} / \mathrm{dt}$ is the rate of increase of the cable length, $\mathrm{L} 0$ is the initial length of the cable unit. Given the solid structure inside the dynamic cable, the inner cross-sectional area and hydrostatic internal pressure of dynamic cable are both 0 . Based on the lumped-mass method, the bending moment of the dynamic cable is expressed as:

$$
M=E I \kappa+\left(\lambda_{t} / 100\right) D_{c} \cdot d \kappa / d t
$$

where EI is the cable bending stiffness, $\lambda t$ is the target bending damping, Dc is the bending critical damping value, and $\mathrm{d} \kappa / \mathrm{dt}$ is the rate of curvature change.

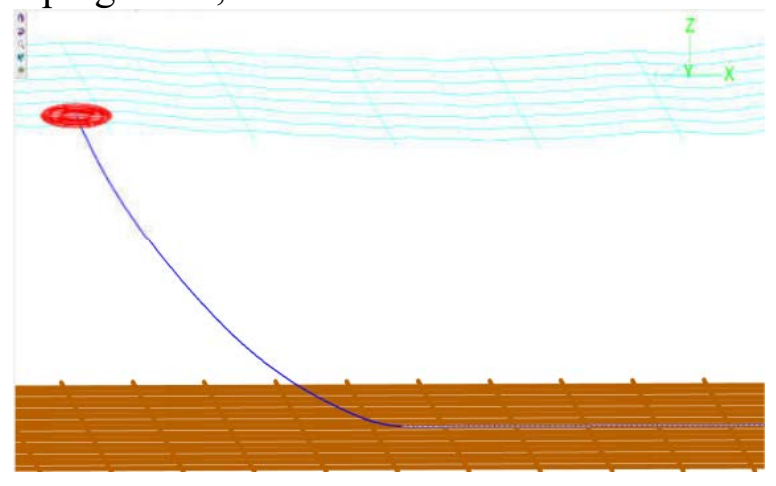

Figure 5. Dynamic cable suspension line arrangement

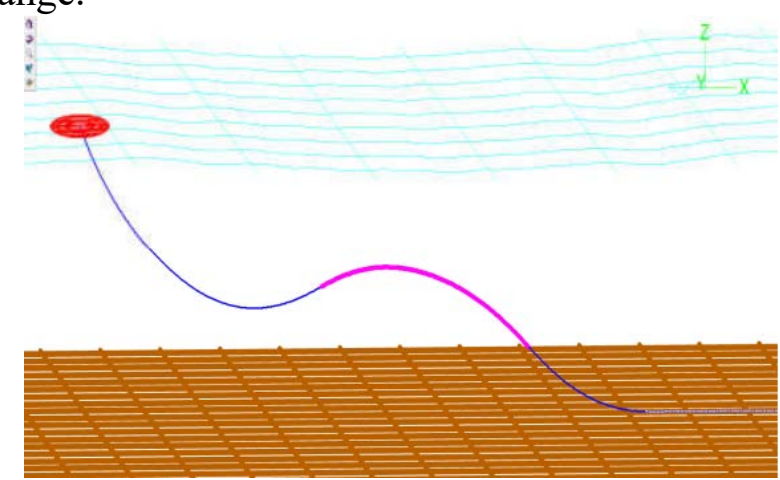

Figure 6. Dynamic cable lazy wave type arrangement

In addition, due to the harsh environment of shallow water, two arrangement configurations, i.e. catenary configuration and lazy wave configuration, are used in this paper to set up the dynamic cable space arrangement for the floating in-rotor type wave energy generator, so the buoyancy block system needs to be introduced. In order to improve the efficiency of the numerical simulation, the riser section with buoyancy blocks (referred to as buoyancy section) is equivalently treated and simplified to a section of riser structure with uniform inner and outer diameters, and the hydrodynamic load per unit length of the buoyancy section and the buoyancy load are equivalent. In addition, the "Vessel" module is selected to simulate the motion of the floating internal rotor wave energy generator. "Time history" module in the "Vessel" module of the OrcaFlex software is used as the input for the top motion response of the dynamic cable. The top connection of the dynamic cable for the wave power generator is set as an articulated connection and the cable anchor point is also articulated. The nonlinear pipesoil model and modified Coulomb friction model are chosen to simulate the pipe-soil vertical and lateral interactions, respectively. In order to improve the computational accuracy of the numerical simulation, the dynamic cable elements in the suspended section and touchdown zone (TDZ) are refined, respectively. Figure 5 and Figure 6 illustrate the OrcaFlex-based finite element models for the dynamic cable catenary configuration and lazy wave configuration, respectively. 


\section{Result discussion and analysis}

Based on water depth of $15 \mathrm{~m}$, the finite element models of catenary dynamic cable and lazy wave dynamic cable are established respectively. In order to facilitate the comparative analysis, the dynamic cable hang-off angles for these two layout configurations are set to $20^{\circ}$ under harsh shallow water condition. The main parameters of dynamic cable are shown in Table 1, and the layout parameters of different configurations are shown in Table 2. Besides, the outer diameter of buoyancy section is $0.1452 \mathrm{~m}$. The current profile is simulated by the power law. The current is positive along the negative $x$-axis direction, and the power law index is set as 7. In addition, the normal stiffness of seabed is 6.0 $\mathrm{kN} / \mathrm{m}^{2}$ [8]. Seawater density of $1025 \mathrm{~kg} / \mathrm{m}^{3}$, and OrcaFlex selected Jonswap spectrum (significant wave height $1.5 \mathrm{~m}$, zero-crossing period $6.0 \mathrm{~s}$ ). The current velocity is $0.88 \mathrm{~m} / \mathrm{s}$. The tangential drag coefficient and normal drag coefficient are 1.2 and 0.008 respectively, and the tangential additional mass coefficient and normal additional mass coefficient are 1.0 and 0.008 respectively [9].

Table 1. Main parameters of dynamic cable

\begin{tabular}{ccc||ccc}
\hline Name & Value & Unit & Name & Value & Unit \\
\hline Outside diameter & 71.7 & $\mathrm{~mm}$ & Weight per unit length in air & 10.5 & $\mathrm{~kg} / \mathrm{m}$ \\
Extensional stiffness & 149.3 & $\mathrm{MN}$ & Minimum tensile load & 990 & $\mathrm{kN}$ \\
Torsional stiffness & 6.0 & $\mathrm{kN} \cdot \mathrm{m}^{2}$ & Maximum working tensile force & 587.5 & $\mathrm{kN}$ \\
Flexural stiffness & 0.52 & $\mathrm{kN} \cdot \mathrm{m}^{2}$ & Minimum bend radius & 1.1 & $\mathrm{~m}$ \\
\hline
\end{tabular}

Table 2. Configuration parameters of different dynamic cables

\begin{tabular}{cccc}
\hline $\begin{array}{c}\text { Dynamic cable layout } \\
\text { configuration }\end{array}$ & $\begin{array}{c}\text { Total } \\
\text { length }\end{array}$ & length & $\begin{array}{c}\text { The starting position of the first buoyancy } \\
\text { section }\end{array}$ \\
\hline Catenary configuration & $150 \mathrm{~m}$ & $/$ & $/$ \\
Lazy wave configuration & $150 \mathrm{~m}$ & $20 \mathrm{~m}$ & $15 \mathrm{~m}$ \\
\hline
\end{tabular}

Note: The length of dynamic cable division element in the suspended section and touchdown zone is $0.1 \mathrm{~m}$, and the length of dynamic cable division element in the flowline is $1.0 \mathrm{~m}$.

Harsh shallow water conditions may lead to extremely significant motion response of wave energy generation device, especially relative to shallow water depth. The motion response of wave energy generation device also has a significant impact on the dynamic response of dynamic cable. Therefore, the traditional catenary layout configuration may lead to the failure destruction of dynamic cable. In this paper, the lazy wave layout configuration is introduced to compare the dynamic response of dynamic cable under and catenary layout configuration. Then, appropriate dynamic cable layout configuration under harsh conditions in shallow water would be selected to ensure the normal operation for the power transmission of the wave energy generation device.

Figure 7 and Figure 8 depict the comparison of static effective tension and static bending curvature for different layout configurations of dynamic cables. By comparison, it can be found that the static effective tension of the lazy wave dynamic cable is much smaller than that of the catenary dynamic cable, which is because the introduction of the buoyancy block system greatly reduces the effective tension of the dynamic cable. The maximum effective tensions of the lazy wave cable and catenary cable occurs at the cable top ( $1.083 \mathrm{kN}$ and $1.680 \mathrm{kN}$ respectively). At the same time, the static bending curvature of the lazy wave dynamic cable is obviously larger than that of the catenary riser, which is due to the negative correlation between the effective tension and static bending curvature of the thin rod when the static analysis of the marine slender rod structure is carried out. The maximum static bending curvature of catenary cable occurs in the cable touchdown zone (TDZ), which is $-0.0745 \mathrm{~m}-$ 1. The maximum static curvature of the lazy wave cable occurs in the arch bending zone of suspended cable, which is $0.117 \mathrm{~m}-1$. 


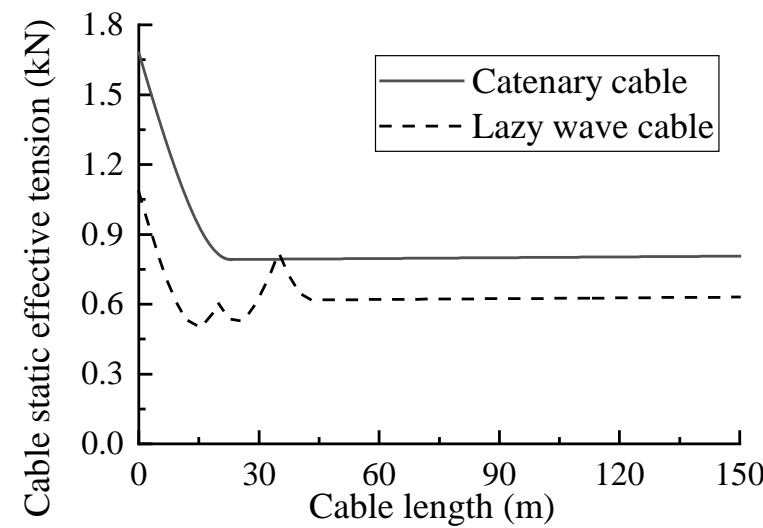

Figure 7. Comparison of static effective tensions of dynamic cable with different configurations

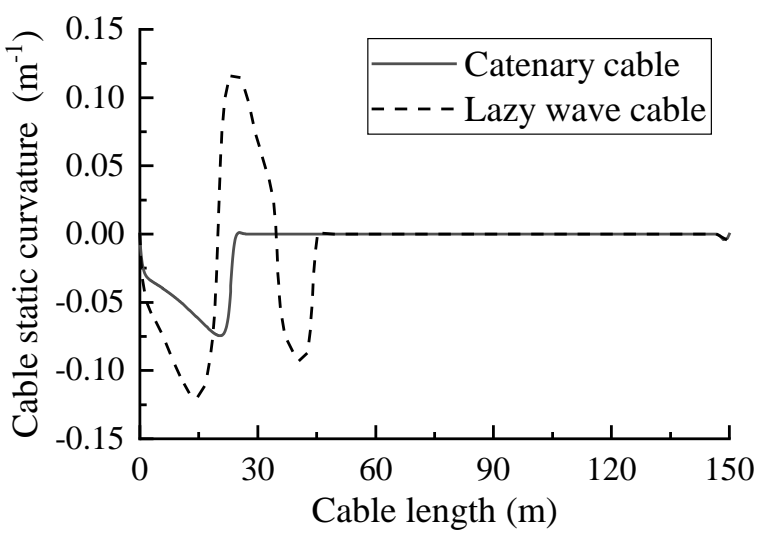

Figure 8. Comparison of static bending cure of dynamic cable with different configurations

Figures 9 11 display the effective tension time-history comparison for the cable top-end, dynamic effective tension comparison and effective tension amplitude comparison with different layout configurations. It can be seen from Figure 9 that the dynamic response of the effective tension at the top-end of the catenary cable is much larger than that of the lazy wave cable. Due to the severe floating body movement caused by the harsh working conditions in shallow water, the compression deformation may even occur at the top-end of the cable. Figure 10 illustrates the maximum effective tension and minimum effective tension of the dynamic cable in the $300 \mathrm{~s}$ numerical simulation time. Through comparison, it is also effectively confirmed that the lazy wave layout configuration can greatly reduce the tension dynamic response of the cable. In addition, the effective tension amplitude of the lazy wave cable is also much smaller than that of the catenary cable, and the effective tension amplitude is the controlling factor of the cable fatigue damage. It can be seen that the lazy wave cable can improve the fatigue life of the dynamic cable by reducing the dynamic effective tension amplitude of the cable.

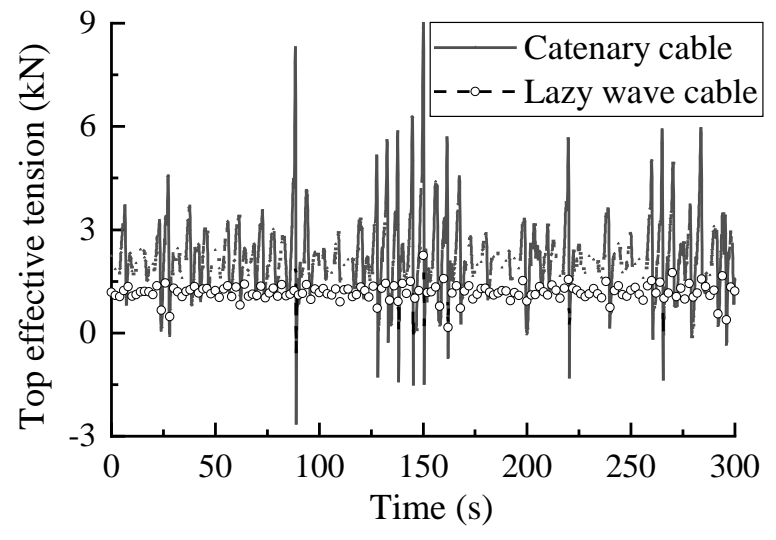

Figure 9. Comparison of effective tensions at the cable top-end with different configurations

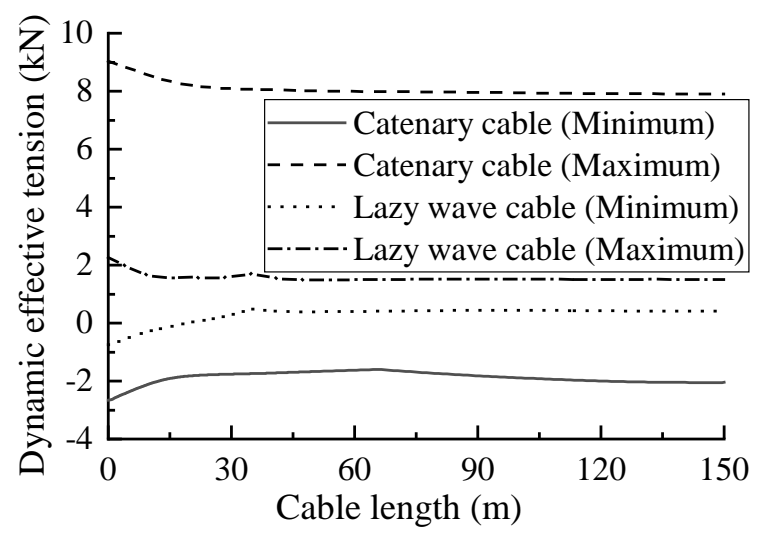

Figure 10. Comparison of dynamic effective tensions of cables with different configurations 


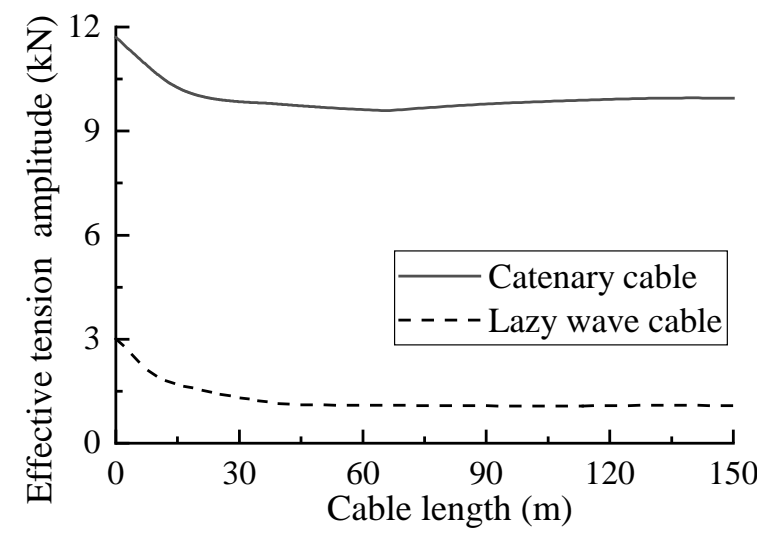

Figure 11. Comparison of dynamic effective tension amplitudes of cables with different configurations

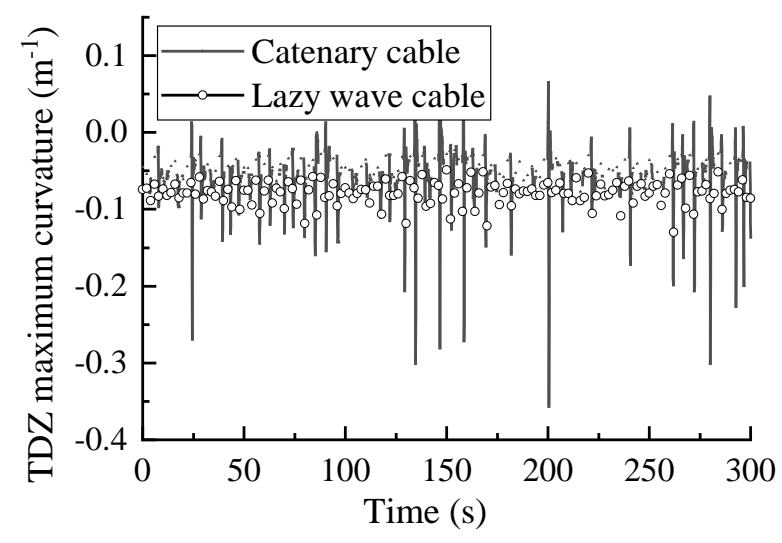

Figure 12. Comparison of maximum curvatures in the cable touchdown zone with different configurations

Figures 12 14 are the TDZ maximum curvature time history comparison, dynamic bending curvature comparison and dynamic curvature amplitude comparison with different layout configurations. It can be seen from Figure 12 that the maximum curvature dynamic response of the catenary cable touchdown zone (TDZ) is significantly higher than that of lazy wave cable. Although the static curvature of the lazy wave cable is larger than that of the catenary cable, the dynamic curvature response of the lazy wave cable will be smaller and smaller than that of the catenary cable as the top floating structure moves more and more significantly (Figure 13). Meanwhile, the dynamic curvature amplitude of catenary cable is much larger than that of lazy wave cable, especially in the hang-off zone and touchdown zone (TDZ). The dynamic curvature amplitude is an important controlling factor of cable fatigue damage, so it can be seen that the fatigue life of dynamic cable can be greatly improved by reducing the dynamic curvature amplitude of the cable.

By comparing the configuration of catenary cable and lazy wave cable, the maximum dynamic effective tensions of catenary cable and lazy wave cable are $9.04 \mathrm{kN}$ and $2.26 \mathrm{kN}$ respectively, which are far less than the maximum working tensile force of cable $587.5 \mathrm{kN}$. The maximum dynamic bending curvatures of catenary cable and lazy wave cable are $-0.358 \mathrm{~m}-1$ and $-0.153 \mathrm{~m}-1$, respectively, which are smaller than the maximum bending curvature of cable $0.909 \mathrm{~m}-1$ (minimum bending radius $1.1 \mathrm{~m}$ ). Considering the complex marine environment, the safety factor of dynamic cable should be set as far greater than 2, so the catenary cable layout configuration does not meet the design requirements under this condition, and the lazy wave type layout configuration would be the suitable dynamic cable layout configuration under shallow water and harsh conditions.

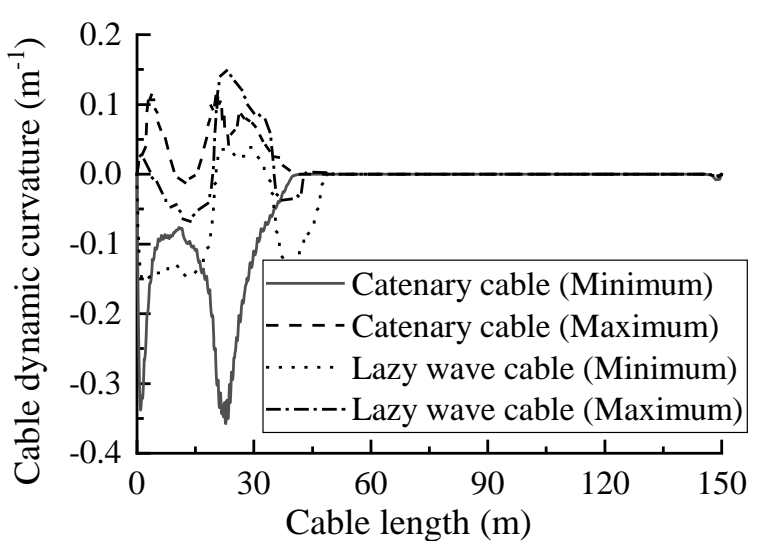

Figure 13. Dynamic curvature comparison of dynamic cables with different configurations

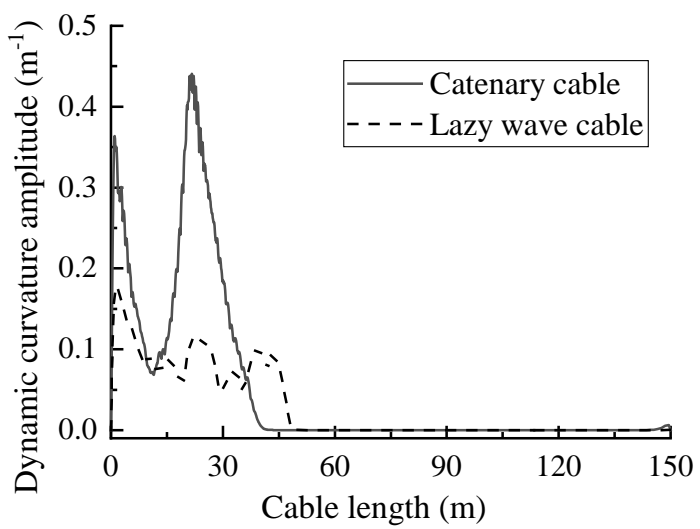

Figure 14. Comparison of dynamic curvature amplitudes of dynamic cables with different configurations 


\section{Conclusions}

In this paper, AQWA module and OrcaFlex software were employed to establish the numerical simulation model of the built-in eccentric rotor wave energy generation device under the constraint of three cables and numerical simulation model of the dynamic cable dynamic response of floating inner rotor wave energy generation device in three-dimensional space. The motion response of the wave energy generation device obtained by the numerical simulation of AQWA module was used as the topend input of the dynamic cable in the OrcaFlex model. The motion responses of different dynamic cable layouts under the harsh conditions of shallow water were compared and studied. It was found that although the static bending curvature of the lazy wave cable is greater than that of the catenary cable under the same working conditions, the dynamic bending curvature of the lazy wave cable is smaller than that of the catenary cable considering that the top-end of the dynamic cable is affected by the motion of the floating inner rotor wave energy generation device. In addition, the static and dynamic effective tensions of lazy wave cable are also less than these of catenary cable.

\section{Acknowledgement}

The authors would like to acknowledge the support of the National Key Research and Development Program of China (2017YFE0115000, 2017YFC0307600 and 2016YFC0303400).

\section{References}

[1] Hongda Shi, Zhen Liu. Research status and development tendency of ocean wave energy[J]. Science \& Technology Review,2021,39(06):22-28.

[2] Heng Tian, Liang Sun, Yu wei Nie, Lin Xu, Liqun Chao. Experimental study on two-body floating point absorber with retractable power take-off[J]. Energy Conservation, 2021, 40(04): 21-24.

[3] Yaqun Zhang, Wensheng Wang, Wanzhen Le, Songwei Sheng. Shape optimization design of hawk wave power generation device[J]. Shipbuilding of China,2021,62(01):80-88.

[4] Shunyuan Xu, Jiaming Wu. Energy Efficiency Analysis of Float Generator Based on AQWA[J]. Shipbuilding of China,2020,61(S2):402-412.

[5] Roy S. Analytical estimates of short duration mean power output and variability for deepwater wave power generation[J]. Energy, 2021, 230.

[6] Mosquera F D, Evangelista C A, Puleston P E, Ringwood J V. Optimal wave energy extraction for oscillating water columns using second-order sliding mode control[J]. IET Renewable Power Generation, 2020, 14(9).

[7] Orcina, 2017. OrcaFlex User Manual, Version 10.2. Cumbria, UK.

[8] Aubeny C P, Shi H, Murff J D. Collapse Loads for a Cylinder Embedded in Trench in Cohesive Soil[J]. International Journal of Geomechanics, 2005, 5(4):320-325.

[9] Weidong Ruan, Bo Sun, Xiaowei Liao. Study on dynamic optimization mechanism of deepwater riser based on lazy-wave configuration[J]. The Ocean Engineering, 2020,38(03):62-74. 